\title{
Silicon Nano-Particles with High Resistance to Harsh Ambient Conditions
}

\author{
Sabri Alkis • Ali K. Okyay
}

Received: 13 December 2011/Published online: 25 April 2012

(C) Springer Science+Business Media, LLC 2012

\begin{abstract}
Silicon nanoparticles which have an average size of $1 \mathrm{~nm}$ are synthesized using electrochemical methods and their stability under high temperature and humidity conditions have been investigated. These types of Silicon nanoparticles exhibit strong blue emission (centered around $420 \mathrm{~nm}$ ) upon excitation with ultraviolet illumination. Standard heating procedures showed that, these nanoparticles in a liquid suspension (de-ionized water) are stable to heating and they retain characteristic emissions even at elevated temperatures. Thin solid films of such Silicon nanocrystals also show good stability under plasma and oxidizing environments at high temperatures.
\end{abstract}

Keywords Silicon - Nanocrystal - Electro-chemical · Plasma stability · High temperature stability $\cdot$ Photoluminescence (PL)

\section{Introduction}

Luminescent Silicon particles have gathered immense interest for application possibilities in solid state lighting, full-color displays, optical sensors and as fluorescent tags for biological imaging. There are different methods for producing $\mathrm{Si}$ nanoparticles reported in the literature. It is possible to obtain Silicon nanoparticles using laser ablation from a solid source, with limited control on the particle size distribution [1, 2]. Chemical vapor deposition (CVD) methods are extensively investigated for producing Silicon nanoparticles [3, 4], resulting in nanoparticles

S. Alkis · A. K. Okyay $(\bowtie)$

Department of Electrical and Electronics Engineering, Bilkent University, Ankara 06800, Turkey e-mail: aokyay@ee.bilkent.edu.tr

S. Alkis - A. K. Okyay

UNAM-National Institute of Materials Science and Nanotechnology, Bilkent University, Ankara 06800, Turkey 
embedded in a host matrix such as $\mathrm{SiO}_{2}$ or $\mathrm{Si}_{3} \mathrm{~N}_{4}$. Such produced particles are then isolated into solution form by selective chemical removal of the host matrix. Since the CVD process is essentially a surface reaction, high volume production of nanocrystals is challenging. Researchers also produced Silicon nanocrystals by ionimplantation of $\mathrm{Si}$ species into $\mathrm{SiO}_{2}$ layers followed by furnace annealing or rapid annealing processes $[5,6]$. Swihart and co-workers reported a process to prepare macroscopic quantities of luminescent Silicon nanoparticles by laser pyrolysis of silane in $\mathrm{He}$ and $\mathrm{H}_{2}$ ambient followed by wet oxidation and etching [7]. This technique initially yields red-orange luminescing particles. Blue luminescing particles are also possible by a post rapid thermal oxidation step. Teo et al. synthesized Silicon nanostructures using thermal evaporation of $\mathrm{SiO}$ powder using oxide assisted growth method. They demonstrate the strong effect of $\mathrm{pH}$ on the stability of $\mathrm{Si}$ nanostructures and they did report an optimum $\mathrm{pH}$ range for the Silicon nanostructures that are obtained using the above method $[8,9]$. In this work, effects of $\mathrm{pH}$ of the solution on stability are not investigated.

Silicon nanoparticles' stability is crucial for successful process integration for exciting applications. Luminescing Silicon particles $(<3 \mathrm{~nm})$ that are free from a substrate (usually in a colloidal suspension) degrade over time mainly via the consumption of Silicon atoms by oxidation [10]. This is a significant issue for all of the preparation methods described above. For instance, Swihart et al [7]. investigate the stability of orange particles in colloidal suspensions at room temperature reporting a significant decay in photoluminescence intensity after several hours in water (several days in alcohol). They reportedly improve the stability of the particles by treatment in a chemical oxidizing ambient $\left(\mathrm{HNO}_{3}\right.$ solution), extending the degradation duration at room temperature [7]. However, there is no investigation about stability of Silicon particles at any harsh ambient conditions beyond room conditions. Also, any investigation of stability of blue particles is lacking.

A recent technique, reported by Nayfeh [11, 12], is a high throughput and low cost method for producing Silicon nanoparticles of diameters less than $3 \mathrm{~nm}$. This method is based on electrochemically etching bulk Silicon wafers using a mixture of hydrofluoric acid (HF), hydrogen peroxide $\left(\mathrm{H}_{2} \mathrm{O}_{2}\right)$ and methanol. Upon electrochemical etching, Silicon wafer becomes porous followed by the collection of Silicon nanoparticles from the surface by ultrasonic treatment.

Nayfeh et al. showed that 1-3 nm size Silicon nanoparticles fluoresce in blue, green and red wavelengths upon excitation with ultraviolet (UV) illumination [13]. Such nanoparticles are easily produced and they have numerous technological applications in addition to the ones listed above. Up to date, efficient UV photodetectors [14], and metal-oxide-semiconductor (MOS) memory devices [15], have been developed and reported in the literature. Researchers also investigated the effect of Silicon nanoparticles on the power conversion efficiency of solar cells. It has been shown that, $1 \mathrm{~nm}$ size (blue fluorescing) particles and $3 \mathrm{~nm}$ size (red luminescent) particles increase the power performance of solar cells up to $60 \%$ in the UV/blue range. In the visible range the power enhancements turned out to be $10 \%$ for the red and $3 \%$ for the blue particles [16].

Inspired by these research results, we prepared Silicon nanoparticles using a similar electrochemical method described by Nayfeh and co-workers [13, 17]. There 
is, to our knowledge, no report about the stability of optical properties of such eletrochemically prepared Silicon nanocrystals. In this work we investigate the effect of heating, humidity and plasma on optical properties of such pure Silicon nanocrystals both in liquid medium and as thin solid films.

\section{Material and Methods}

Silicon nanoparticles were obtained through anodization of bulk $p$-type Silicon wafer $(0.01 \mathrm{ohm} \mathrm{cm})$ in a mixture of $\mathrm{H}_{2} \mathrm{O}_{2} / \mathrm{HF}$ and methanol. A standart Keithley 2400 sourcemeter was used to maintain constant current $\left(1-20 \mathrm{~mA} / \mathrm{cm}^{2}\right)$ in the etching setup. After several hours $(1-10 \mathrm{~h})$ of etching process, the wafer was transferred to a glass vial filled with de-ionized (DI) water and the solution was sonificated with a commercial ultrasound bath. Large particles dislodged by the sonification were filtered out using a commercial $100 \mathrm{~nm}$ filter paper (Minisart High-Flow, 16553). Transmission electron microscopy (TEM) images of Silicon nanoparticles were taken using FEI Technai, G2 F30 type of electron microscope after drop casting the samples from solution on a $\mathrm{Cu}$ grid. Figure $1 \mathrm{a}-\mathrm{d}$ shows 1-3 nm size crystalline Silicon nanoparticles. Particle size distribution and average particle size was obtained by a Malvern, Nano ZS type Zeta potential instrument. The average size of our Silicon nanoparticles determined by Zeta-Sizer instrument was $\sim 1 \mathrm{~nm}$.

Stability of Silicon nanocrystals in both colloidal suspensions in liquid and thin film forms were investigated. For all cases, photoluminescence (PL) intensities were monitored after each treatment with a VARIAN (Cary Eclipse) spectrofluorometer instrument. Throughout the analysis presented here, there was no attempt made to separate particles of different sizes, which could be very challenging for particles of sizes such as ones that are reported in the manuscript (several nanometers) and deserve a separate investigation. Measurements were done on the solutions with a distribution of various size particles as shown in TEM images. We did not study the effect of size on the stability of electrochemically obtained Silicon nanoparticles.

\section{Results and Discussions}

\section{Stability of Silicon Nanocrystals in Liquid Medium}

In order to investigate the stability of Silicon nanocrystals in aqueous medium, Silicon crystals in DI water are collected inside a glass vial and heated using a commercial heater at the boiling temperature of water $\left(100{ }^{\circ} \mathrm{C}\right)$. The colloidal solutions were heated consecutively for $15 \mathrm{~min}, 1 \mathrm{~h}$ and $3 \mathrm{~h}$ at $100{ }^{\circ} \mathrm{C}$, and $\mathrm{PL}$ measurements were obtained upon excitation with $250 \mathrm{~nm}$ and $300 \mathrm{~nm}$ wavelength photons. $300 \mathrm{~nm}$ excitation gave a broader PL emission compared to $250 \mathrm{~nm}$ excitation. Recorded PL intensities for $250 \mathrm{~nm}$ and $300 \mathrm{~nm}$ excitation are plotted in Fig. 2a and b, respectively. $300 \mathrm{~nm}$ excitation gave a broader PL emission compared to $250 \mathrm{~nm}$ excitation. We think that, photons with different energies 


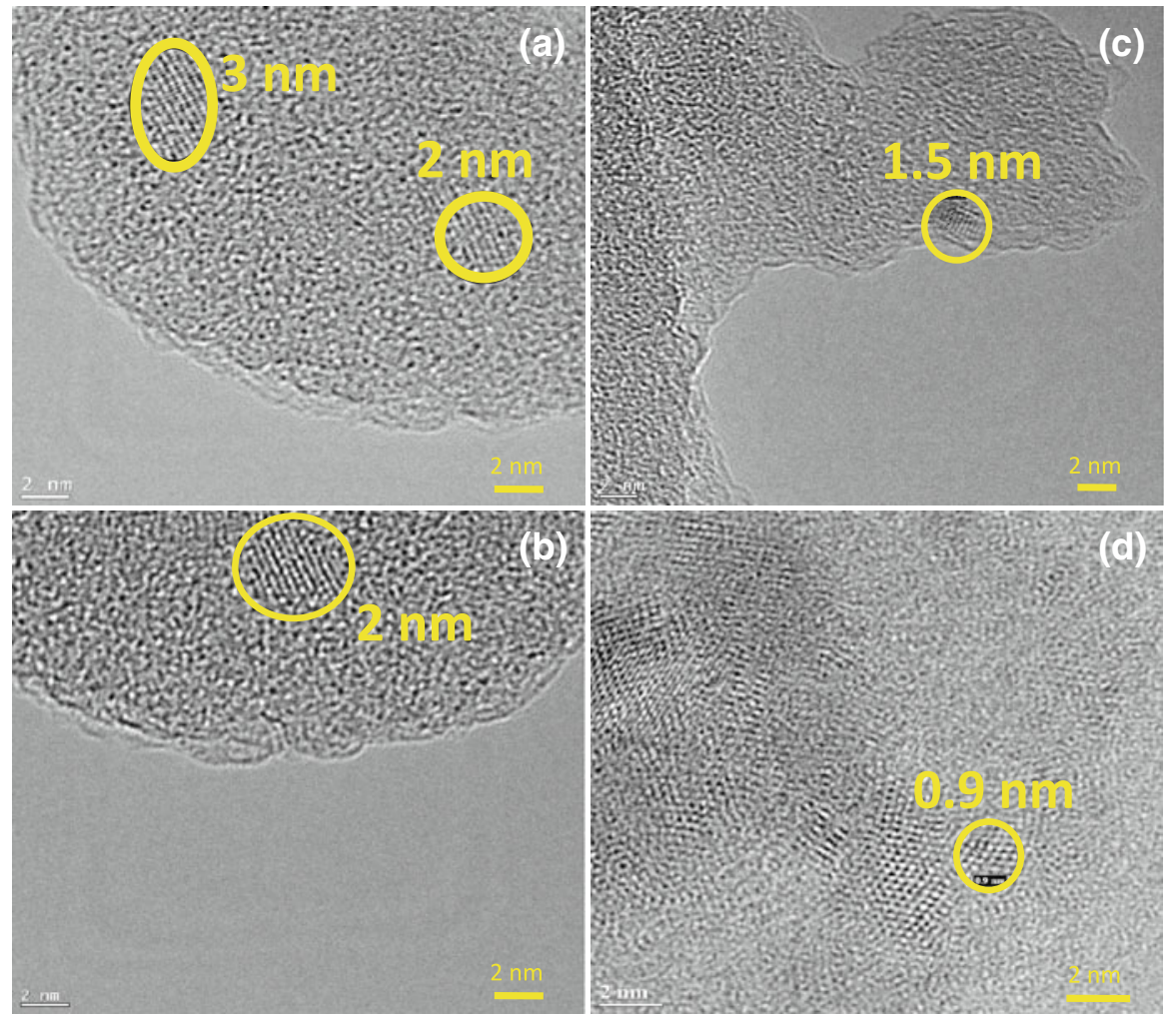

Fig. 1 Transmission electron microscope image of particles of size. a $2 \mathrm{~nm}$ and $3 \mathrm{~nm}, \mathbf{b} 2 \mathrm{~nm}, \mathbf{c} 1.5 \mathrm{~nm}$, d $0.9 \mathrm{~nm}$

excite electrons from the valence band to different states in the conduction band which lead to different relaxation patterns. We believe that, this accounts for different PL emissions upon different wavelength excitations. Particles obtained by the above described method are shown to be Hydrogen $(\mathrm{H}-)$ terminated $[16,18]$. The blue-luminescence from such small $(\sim 1 \mathrm{~nm})$ particles is attributed to the presence of Hydrogen $(\mathrm{H})$ bonds (i.e. H-termination) [10]. Friesner et. al. show that Oxygen terminated nanocrystals of such sizes characteristically luminesce at yellow-red colors [10]. The lack of any observable change in the spectrum and the characteristic blue luminescence is, attributed to the stable H-termination of Silicon nanoparticles compared to fast decrease in luminescence efficiency of such particles produced by other techniques even at room temperature [7].

Stability of Thin Film Silicon Nanocrystals

Thin solid films of Silicon nanocrystals were prepared by dropcasting nanocrystal colloidal solution on a quartz substrate followed by evaporating DI water via heating at $50{ }^{\circ} \mathrm{C}$. In order to investigate stability of nanoparticles to harsh conditions 
Fig. 2 Photoluminescence characteristics of initial and heated Si nanocrystals in DI water excited with a $250 \mathrm{~nm}$ and b $300 \mathrm{~nm}$ illumination
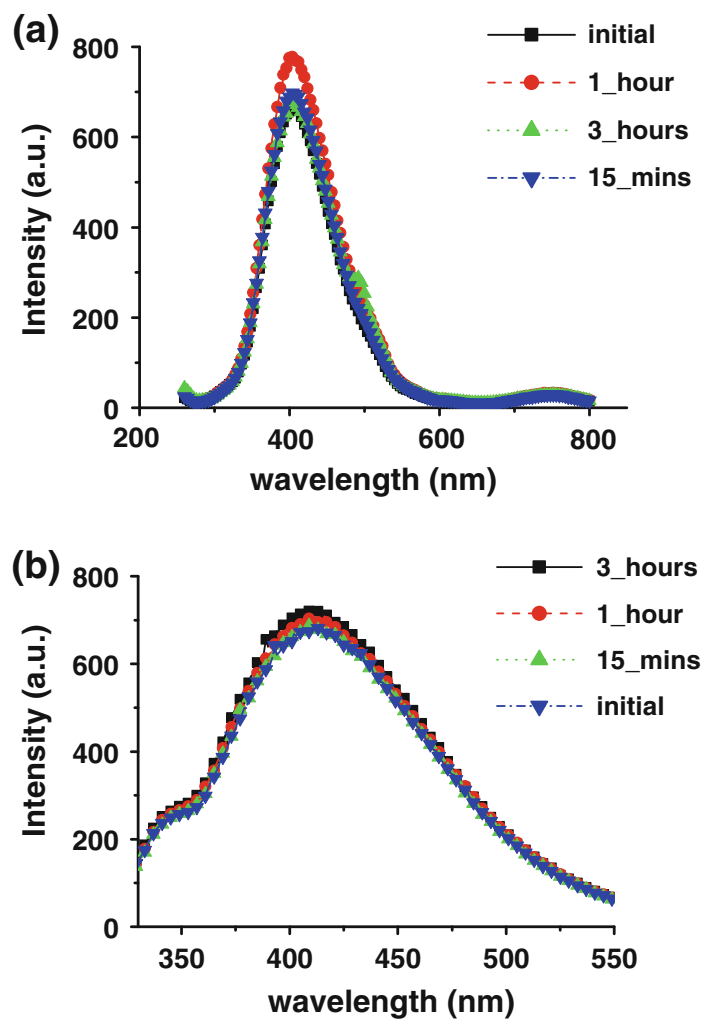

such as oxygen plasma, we used plasma enhanced chemical vapor deposition (PECVD) tool and commercial plasma asher system (NANOPLAS, DSB 6000) available at UCF (UNAM cleanroom facilities). Since thin films were prepared by dropcasting, nanocrystal concentration on quartz substrate surface was non-uniform. Consecutive PL measurements were conducted after a best effort to focus the excitation beam on the same spot each time.

\section{PECVD Treated Thin Solid Films}

Thin film samples were treated at $250{ }^{\circ} \mathrm{C}$ (the highest available with this tool) with an $\mathrm{O}_{2}$ flux of $50 \mathrm{sccm}$ (standard cubic centimeter) and an RF power of $9 \mathrm{~W}$. Figure $3 a$ and $b$ show PL data obtained from an initial (untreated) sample and from a sample which was treated with PECVD tool for $2 \mathrm{~h}$, at $250 \mathrm{~nm}$ and $270 \mathrm{~nm}$ excitation, respectively. PL measurements were obtained using a $2.5 \%$ attenuator in order to prevent the oversaturation of the emission peaks. Thin solid samples show emissions at blue wavelengths similar to the liquid samples, however PL measurements from solid samples exhibit additional peaks which we believe are due to strong reflection of the second harmonic of the excitation source beam. These peaks are highlighted as "second order harmonics" on the figures. The results show that the spectral emission characteristics of Silicon nanocrystals remain unchanged 
Fig. 3 Photoluminescence characteristics of initial and PECVD-treated Si nanocrystals in thin film form excited with a $250 \mathrm{~nm}$ and b $270 \mathrm{~nm}$ illumination
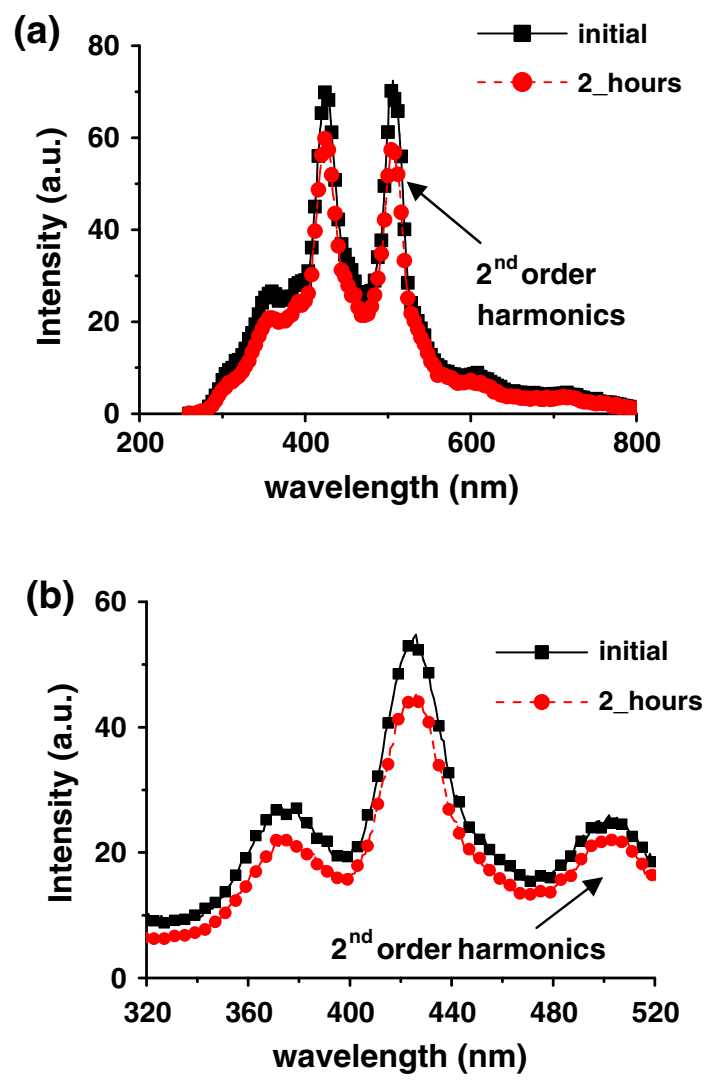

for significant blue particles, an indication of presence of H-termination. The slight decrease in PL intensity is attributed to non-uniform concentration of nanoparticles on the substrate due to drop casting deposition.

\section{Asher Treated Thin Solid Films}

Prepared thin film samples were treated at $180{ }^{\circ} \mathrm{C}$ with an $\mathrm{O}_{2}$ flux of $50 \mathrm{sccm}$ and RF power of 9 watts, inside the Nanoplas asher tool. Figure $4 \mathrm{a}$ and $\mathrm{b}$ show PL data obtained from an untreated sample and from a sample treated with the asher for $2 \mathrm{~h}$, at $250 \mathrm{~nm}$ and $270 \mathrm{~nm}$ excitation, respectively. Once again, PL measurements were obtained using a $2.5 \%$ attenuator in order to prevent the oversaturation of the emission peaks. Similar to PECVD treatment, the consistent spectral PL characteristics and the strong component in the blue wavelength indicate H-termination and lack of $\mathrm{Si}-\mathrm{O}$ bonds. The slight increase in PL intensity is again attributed to non-uniform concentration of Silicon nanoparticles due to drop casting.

It has been reported in the literature that, these types of electrochemically obtained Silicon nanoparticles are spherical and they have two shell structures (outer shell and inner shell). They have an outer shell which has fullerene like 
Fig. 4 Photoluminescence characteristics of initial and asher-treated Si nanocrystals in thin film form excited with a $250 \mathrm{~nm}$ and b $270 \mathrm{~nm}$ illumination
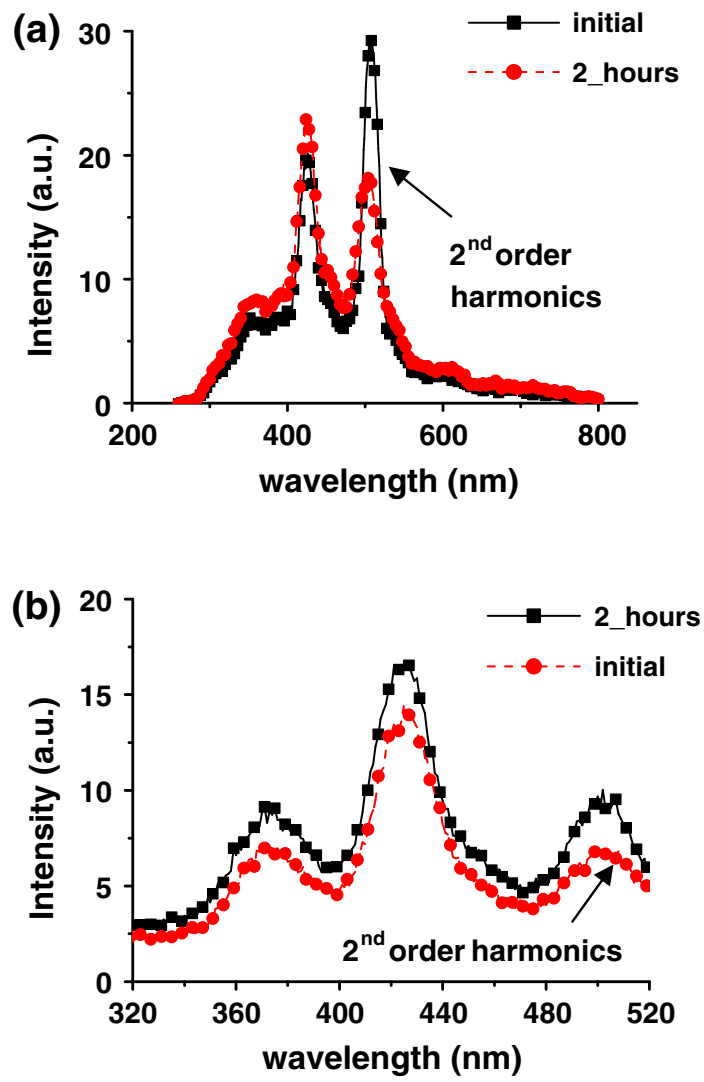

topology and the inner shell has a high coordination number (see Ref. 19). We believe that, this structure accounts for the high stability of these nanoparticles. Since the PL spectrum does not red shift upon treatment, we beleive that $\mathrm{H}$-termination is maintained $[6,7]$.

\section{Conclusions}

Stability of electrochemically prepared Silicon nanocsrystals is investigated in liquid form by thermal heating in aqueous solution. Also, the effect of high temperature oxygen plasma on luminescence characteristics of such particles is investigated. Ultra-small $(\sim 1 \mathrm{~nm})$ particles prepared by anodization of Si wafers are extremely durable at harsh conditions, required for potential process integration, verified by the spectral photoluminescence characteristics. The stability of such nanoparticles are attributed to the fullerene like outer shell and the highly coordinated inner shell structure.

Acknowledgments This work was supported by TUBITAK 108E163, 109E044, EU FP7 PIOS 239444. The authors gratefully acknowledge TUBITAK-BIDEB. 


\section{References}

1. Y. Nakata, J. Muramoto, T. Okada, and M. Maeda (2002). J. Appl. Phys. 91, 1640-1643.

2. D. B. Geohegan, A. A. Puretzky, G. Duscher, and S. J. Pennycook (1998). Appl. Phys. Lett. 72, 2987-2989.

3. K. Nakaso, B. Han, K. H. Ahn, M. Choi, and K. Okuyama (2003). J. Aerosol Sci. 34, 869-881.

4. S. Dusane, T. Bhave, S. Hullavard, S. V. Bhoraskar, and S. Lokhare (1999). Solid State Commun. 111, 431-435.

5. T. Shimizu-Iwayama, T. Hama, D. E. Hole, and I. W. Boyd (2005). Nucl. Instrum. Methods Phys. Res. B 230, 203-209.

6. T. Shimizu-Iwayama, N. Kurumado, D. E. Hole, and P. D. Townsend (1998). J. Appl. Phys. 83, 6018-6022.

7. X. Li, Y. He, S. S. Talukdar, and M. T. Swihart (2003). Langmuir 19, 8490-8496.

8. W. W. Chen, X. H. Sun, S. D. Wang, S. T. Lee, and B. K. Teo (2005). J. Phys. Chem. B 109, 10871-10879.

9. B. K. Teo, W. W. Chen, X. H. Sun, S. D. Wang, and S. T. Lee (2005). J. Phys. Chem. B 109, $21716-21724$

10. Z. Zhou, L. Brus, and R. Friesner (2003). Nano Lett. 3, 163-167.

11. O. Akcakir, J. Therrien, G. Belomoin, N. Barry, J. D. Muller, E. Gratton, and M. Nayfeh (2000). Appl. Phys. Lett. 76, 1857-1859.

12. L. Mitas, J. Therrien, R. Twesten, G. Belomoin, and M. H. Nayfeh (2001). Appl. Phys. Lett. 78, 1918-1920.

13. E. Rogozhina, G. Belomoin, A. Smith, L. Abuhassan, N. Barry, O. Akcakir, P. V. Braun, and M. H. Nayfeh (2001). Appl. Phys. Lett. 78, 3711-3713.

14. O. M. Nayfeh, S. Rao, A. Smith, J. Therrien, and M. H. Nayfeh (2004). IEEE Photonics Technol. Lett. 16, 1927-1929.

15. O. M. Nayfeh, D. A. Antoniadis, K. Mantey, and M. H. Nayfeh (2007). Appl. Phys. Lett. 90, 153105-153107.

16. M. Stupca, M. Alsalhi, T. Al Saud, A. Almuhanna, and M. H. Nayfeh (2007). Appl. Phys. Lett. 91, 063107-063109.

17. M. Nayfeh, O. Akcakir, J. Therrien, Z. Yamani, N. Barry, W. Yu, and E. Gratton (1999). Appl. Phys. Lett. 75, 4112-4114.

18. S. Rao, K. Mantey, J. Therrien, A. Smith, and M. Nayfeh (2007). Phys. Rev. B 76, 155316.

19. G. Belomoin, J. Therrien, A. Smith, S. Rao, R. Twesten, S. Chaieb, M. H. Nayfeh, L. Wagner, and L. Mitas (2002). Appl. Phys. Lett. 80, 841-843. 